\title{
Eddy Current Testing Using Square-wave Inverter for Thickness Inspection of Steel Plate
}

\author{
Teruyoshi Sasayama* and Keiji Enpuku \\ Department of Electrical Engineering, Kyushu University, \\ 744 Motooka, Nishi-ku, Fukuoka 819-0395, Japan
}

(Received January 31, 2019; accepted April 11, 2019)

Keywords: eddy current testing, low frequency, nondestructive testing square-wave inverter, steel plate

Eddy current testing (ECT) usually employs a sinusoidal current that flows through an excitation coil. If a square wave instead of a sinusoidal wave is used for excitation, i.e., a square-wave inverter instead of linear amplifiers is used for an ECT system, a handheld ECT system can be developed and the cost can be reduced. In this study, we developed a lowfrequency ECT (LF-ECT) system with a square-wave inverter to determine whether an inverter is applicable to estimating the thickness of a thick steel plate in the range from 6 to $19 \mathrm{~mm}$. The developed ECT system has a differential excitation coil and a pickup coil, and these coils are arranged on the steel plate. A square-wave voltage is applied to the excitation coil, and the voltage of the pickup coil is measured. Subsequently, the mutual equivalent resistance and inductance are calculated. Results indicate that the fundamental component of the resistance increases with the thickness when the frequency is approximately $4 \mathrm{~Hz}$. Furthermore, we determined whether the harmonic signals generated by the inverter are also useful. Results indicate that the harmonic components of the resistance also tend to increase with a sufficiently small increase in thickness, i.e., $\leq 12 \mathrm{~mm}$. This implies that surface and back-surface defects can be simultaneously obtained.

\section{Introduction}

Maintaining industrial infrastructures, such as roads, bridges, tunnels, and buildings, built during the period of rapid economic growth has become a problem because half a century has passed since then. Additionally, developed countries, including Japan, will become full-fledged aged societies in the future, and the shortage of maintenance personnel is a significant concern. Therefore, accurate, easy, and fast inspection methods are required.

These infrastructures are made of steel. Therefore, eddy current testing (ECT), which is one of the nondestructive testing techniques, is promising for detecting the defects of a metallic structure because it can be conducted at high speeds without the specimen coming in contact with the sensor using a compact instrument with no usage restrictions. ${ }^{(1,2)}$ However, conventional ECT is limited to the detection of surface or subsurface defects, owing to the

*Corresponding author: e-mail: sasayama@sc.kyushu-u.ac.jp

https://doi.org/10.18494/SAM.2019.2318 
skin effect. Moreover, steel plates with a thickness of $10 \mathrm{~mm}$ or larger are often used for large infrastructures. Therefore, low-frequency ECT (LF-ECT) is essential for examining the defects of a thick ferromagnetic object. ${ }^{(3-9)}$

ECT usually employs a sinusoidal current that flows through an excitation coil. ${ }^{(1,2)}$ In this case, linear amplifiers are used to generate the excitation current. However, if a large excitation current is required to enhance the signal from the eddy current, a linear amplifier requires a large heat sink owing to the low conversion efficiency, and the weight and size of the ECT system increase, which lowers its portability. On the other hand, electrical equipment such as motors often employs an inverter to generate a large current owing to development of power electronics. An inverter is more efficient, smaller, and lighter than a linear amplifier, which contributes to the development of a handheld ECT system and cost reduction.

We previously developed an LF-ECT system using a high-temperature superconducting (HTS) coil and a pulse width modulation (PWM) inverter. ${ }^{(9)}$ Using this system, a steel plate thickness of up to $20 \mathrm{~mm}$ can be estimated by measuring the change in coil resistance even when the liftoff is approximately $100 \mathrm{~mm}$, which contributes to the detection of the back-surface corrosion of a thick steel plate.

An inverter is also considered to be applicable to LF-ECT with copper coils as well as HTS coils. In this study, we determine whether an inverter is applicable to LF-ECT with copper coils to estimate the thickness of a steel plate. Note that a square wave contains harmonic sinusoids. Therefore, we also determine whether more information can be obtained simultaneously from harmonic sinusoids as well as a fundamental sinusoid. To investigate the efficacy of harmonic sinusoids, we apply a square wave inverter instead of a PWM inverter.

\section{Materials and Methods}

Figure 1 shows the developed LF-ECT system with a square-wave inverter. Firstly, a stable DC voltage $(5 \mathrm{~V})$ was generated by a DC/DC converter. Then, the square wave was generated by an H-bridge driver (BD6212HFP, ROHM Corp., Ltd.) to excite the coil. The sum of the resistances of the upper and lower arms is $0.5 \Omega$ (typical value). A shunt with a resistance of $0.1 \Omega$ was inserted to measure the current of the excitation coil.

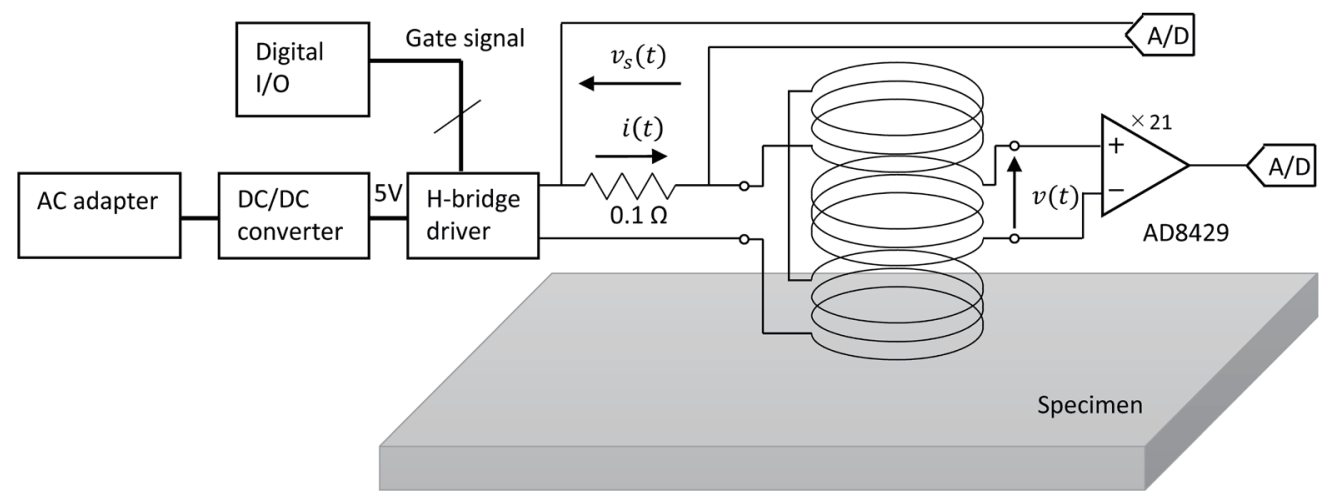

Fig. 1. LF-ECT system with an inverter. 
The gate signal of the H-bridge driver was generated by a digital I/O module (NI 9401, National Instruments Corp.). Using the I/O module, we generated a square wave whose frequency was $f \mathrm{~Hz}$. The voltage of the pickup coil $v(t)$ was amplified and recorded by an A/D converter (National Instruments Corp., NI 9239; resolution, 24 bit; sampling rate, $50 \mathrm{kS} / \mathrm{s}$ ). The digital I/O module and the A/D converter were set in the CompactDAQ chassis (cDAQ-9171, National Instruments Corp.), and the modules were controlled using the LabVIEW software.

The lower and upper excitation coils were connected reversely to cancel out the direct flux from the excitation coils and to detect only the flux generated by an eddy current. The voltage of the shunt resistance as well as the pickup coil was recorded by the A/D converter, and the excitation current $i(t)$ was obtained. Subsequently, the $n$-th harmonic voltage $\dot{V}_{n}$ and current $\dot{I}_{n}$ were calculated by applying a fast Fourier transform (FFT) to $v(t)$ and $i(t)$, respectively.

When we assume that the magnetic field is small enough to ignore the nonlinearity and hysteresis characteristics of the steel plate, the relationship between $\dot{V}_{n}$ and $\dot{I}_{n}$ can be described as

$$
\dot{V}_{n}=\left(\Delta R_{n}^{M}+j n \omega \Delta M_{n}\right) \dot{I}_{n}
$$

where $\omega$ is the angular frequency $(\omega=2 \pi f), R_{n}^{M}$ denotes the apparent resistance due to the magnetic flux generated by the eddy current, or equivalent resistance, $M_{n}$ denotes the mutual inductance between the excitation and pickup coils, and $\Delta$ denotes the difference between the values obtained with and without the plate. The parameters $\Delta R_{n}^{M}$ and $\Delta M_{n}$ were obtained by measuring $\dot{V}_{n}$ and $\dot{I}_{n}$.

Figure 2 shows the arrangement of the excitation and detection coils. Table 1 shows the resistances and inductances as well as the dimensions of these coils. These resistance and inductances were measured using an LCR meter (ZM2371, NF Corp.). The distance between the specimen and the lower excitation coil, i.e., lift-off, was $3 \mathrm{~mm}$.

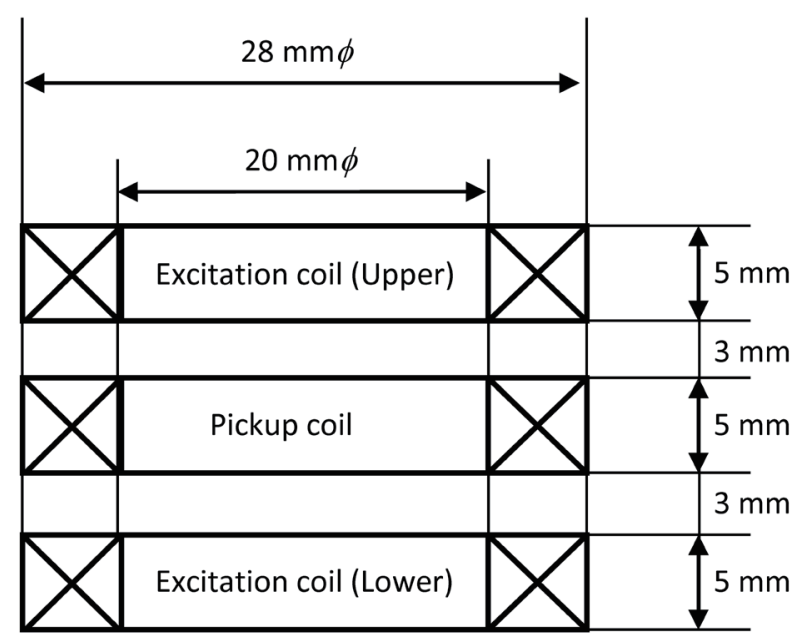

Fig. 2. Arrangement of the excitation and pickup coils. 
Table 1

Specifications of the excitation and pickup coils.

\begin{tabular}{lccc}
\hline & Pickup coil & Upper excitation coil & Lower excitation coil \\
\hline Inner diameter $(\mathrm{mm})$ & 20 & 20 & 20 \\
Outer diameter $(\mathrm{mm})$ & 28 & 28 & 28 \\
Number of turns & 1200 & 1200 & 1200 \\
Resistance $(\Omega)$ & 200.6 & 199.5 & 200.6 \\
Inductance $(\mathrm{mH})$ & 40.89 & 40.94 & 41.18 \\
\hline
\end{tabular}

The square voltage wave can be described as

$$
e(t)=\left\{\begin{array}{cc}
-E, & -\frac{T}{2} \leq t<0, \\
E, \quad 0 \leq t<\frac{T}{2},
\end{array}\right.
$$

where $T$ is the period $(T=1 / f)$ and $E$ is the amplitude $(E=5.0 \mathrm{~V})$. By calculating Fourier series coefficients of $e(t)$, we obtain the equation

$$
e(t)=\frac{4 E}{\pi}\left(\sin \omega t+\frac{1}{3} \sin 3 \omega t+\frac{1}{5} \sin 5 \omega t+\cdots\right) .
$$

Therefore, the $n$-th harmonic voltage of $e(t), E_{n}$, can be derived as

$$
\dot{E}_{n}=\frac{2 \sqrt{2} E}{n \pi}, n=1,3,5, \ldots
$$

Equation (4) implies that a square wave contains rich harmonics. From Eqs. (1) and (4), we obtain the equation

$$
\dot{I}_{n}=\frac{\dot{E}_{n}}{R+j n \omega L}=\frac{2 \sqrt{2} E}{n \pi(R+j n \omega L)}, n=1,3,5, \ldots
$$

where $R$ and $L$ denote the resistance and inductance of the excitation coils, respectively. Here, we assume that $R$ and $L$ are sufficiently larger than the changes in these parameters caused by the eddy current. If $R \gg j n \omega L$, i.e., $n$ and $\omega$ are sufficiently small, $\dot{I}_{n}$ is proportional to $1 / n$. In this study, the parameters $R$ and $L$ are $400.1 \Omega$ and $71.09 \mathrm{mH}$, respectively. Thus, the relation $R \gg n \omega L$ holds when $n f \ll 896$.

In the experiment, SM490A steel plates were used. The relative permeability $\mu_{r}$ is approximately 200, and the conductivity $\sigma$ is $4.1 \mathrm{MS} / \mathrm{m}$. The skin depth $\delta$ is

$$
\delta=\sqrt{\frac{1}{\pi f \sigma \mu_{r} \mu_{0}}}
$$

where $\mu_{0}$ is the permeability of vacuum. Substituting $f=8$ into Eq. (6), we obtained $\delta=$ 
$6.2 \mathrm{~mm}$. We used six plates of different thicknesses, i.e., $d=6,9,12,16,19$, and $22 \mathrm{~mm}$. The dimensions of the steel plates were $200 \times 200 \mathrm{~mm}^{2}$.

\section{Results}

Figure 3 shows the time waveforms of $v(t)$ and $i(t)$ when $f=1 \mathrm{~Hz}$ [Figs. 3(a) $-3(\mathrm{c})$ ] and $f=$ $10 \mathrm{~Hz}$ [Figs. 3(d)-3(f)].

Figures 3(a)-3(c) show the results obtained when $d=0$ (no plate), 6 , and $22 \mathrm{~mm}$, respectively. The amplitude obtained when $d=0 \mathrm{~mm}$ is approximately $1 \mathrm{~V}$, and those obtained when $d=6$ and $22 \mathrm{~mm}$ are approximately $4 \mathrm{~V}$. Therefore, the effect of the steel plate can be determined by comparing the obtained amplitudes. In contrast, the amplitudes fluctuate, and there is no correlation between the amplitude and $d$, e.g., the amplitude obtained when $d=6 \mathrm{~mm}$ is less than that obtained when $d=22 \mathrm{~mm}$. Thus, the thickness $d$ cannot be accurately estimated by measuring only the amplitude.
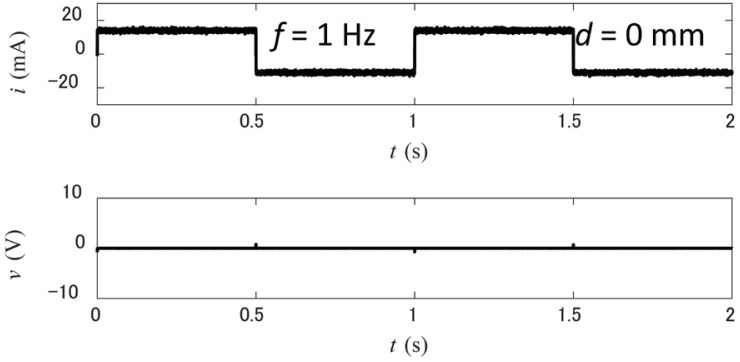

(a)
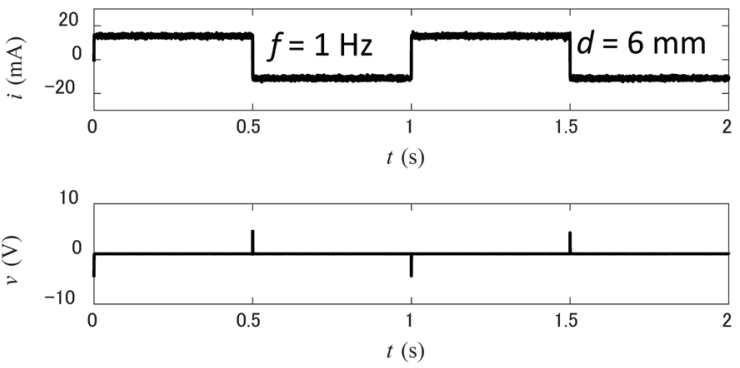

(b)
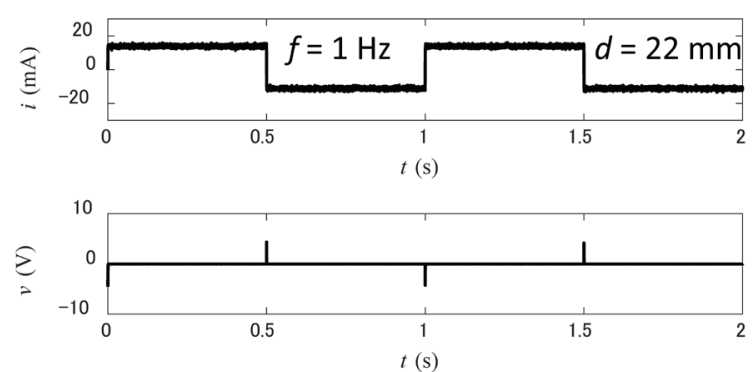

(c)
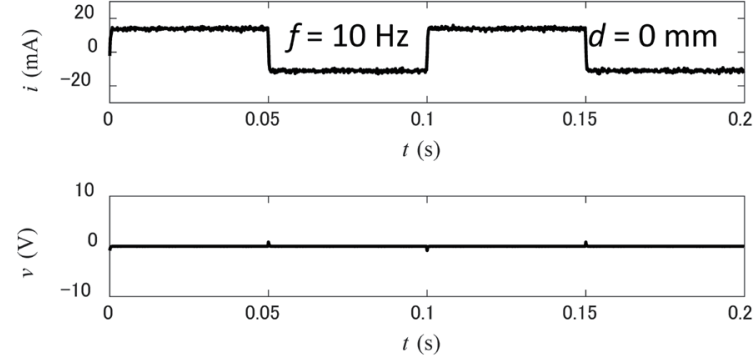

(d)
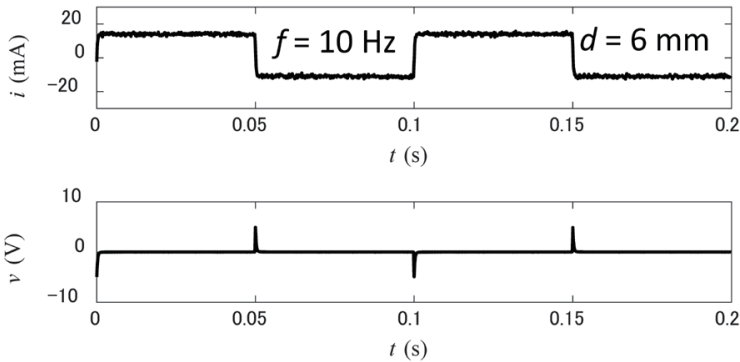

(e)
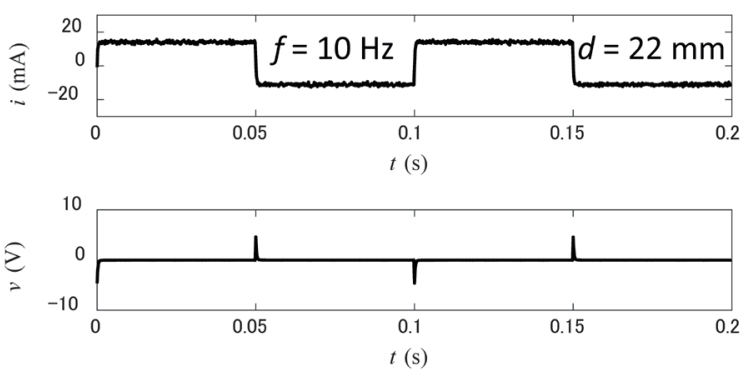

(f)

Fig. 3. Time waveforms of $v(t)$ and $i(t)$. (a) - (c) $f=1 \mathrm{~Hz}$ and (d)-(f) $f=10 \mathrm{~Hz}$. In the upper row, (a), (d), $d=0 \mathrm{~mm}$, in the middle row, (b), (e), $d=6 \mathrm{~mm}$, and in the lower row, (c), (f) $d=22 \mathrm{~mm}$. 
Figures 3(d)-3(f) show the results obtained when $d=0$ (no plate), 6 , and $22 \mathrm{~mm}$, respectively. Here, the tendency observed when $f=10 \mathrm{~Hz}$ is almost the same as that observed when $f=$ $1 \mathrm{~Hz}$. That is, the effect of the steel plate can be determined; however, the thickness cannot be accurately estimated by measuring only the amplitude.

Figure 4(a) shows the relationship between the fundamental mutual equivalent resistance $\left(\Delta R_{1}^{M}\right)$ and the thickness of the steel plate $(d)$ when $\mathrm{f}$ changes from 1 to $10 \mathrm{~Hz}$. Here, $\Delta R_{1}^{M}$ increases with $f$. Thus, it is difficult to recognize the tendency of the result when $f$ is small. When we assume that the hysteresis loss in the steel plate is sufficiently lower than the eddy current loss and the skin effect is small, $\Delta R_{1}^{M}$ is proportional to $f^{2}$.

Figure 4(b) shows the result obtained when $\Delta R_{1}^{M}$ is normalized by dividing $f^{2}$. Here, $\Delta R_{1}^{M}$ monotonically increases with $d$ when $f=3 \mathrm{~Hz}$. Therefore, the thickness $d$ can be estimated by measuring $\Delta R_{1}^{M}$ even when a square wave is used for excitation instead of a sinusoidal wave. In other words, an inverter is applicable to ECT to estimate the thickness of the steel plate.

If the frequency is lower, i.e., $f=1$ and $2 \mathrm{~Hz}$, the signal from the eddy current becomes smaller. Thus, $\Delta R_{1}^{M}$ did not monotonically increase with $d$ when $f=1$ and $2 \mathrm{~Hz}$. That is, it is difficult to estimate $d$ when $f$ is lower. In contrast, if the frequency is higher, i.e., $f \geq 4 \mathrm{~Hz}$, the domain that shows a monotonic increase decreases owing to the skin effect. For example, $\Delta R_{1}^{M}$ monotonically increases in the domain $6 \leq d \leq 12 \mathrm{~mm}$ when $f=10 \mathrm{~Hz}$.

Figure 5 shows the relationship between the fundamental mutual inductance $\left(\Delta M_{1}\right)$ and $d$ when $f$ changes from 1 to $10 \mathrm{~Hz}$. Here, $\Delta M_{1}$ is almost constant even when $d$ and $f$ change. Thus, $\Delta M_{1}$ is independent of $d$.

Figure 6 shows the relationship between the fundamental mutual inductance $\left(\Delta M_{1}\right)$ and $d$ when the lift-off $z$ changes from 3 to $13 \mathrm{~mm}$. Here, $\Delta M_{1}$ is almost constant even when $d$ and $f$ change, whereas $\Delta M_{1}$ decreases with $z$. This suggests that the lift-off can be determined by measuring $\Delta M_{1}{ }^{(8)}$

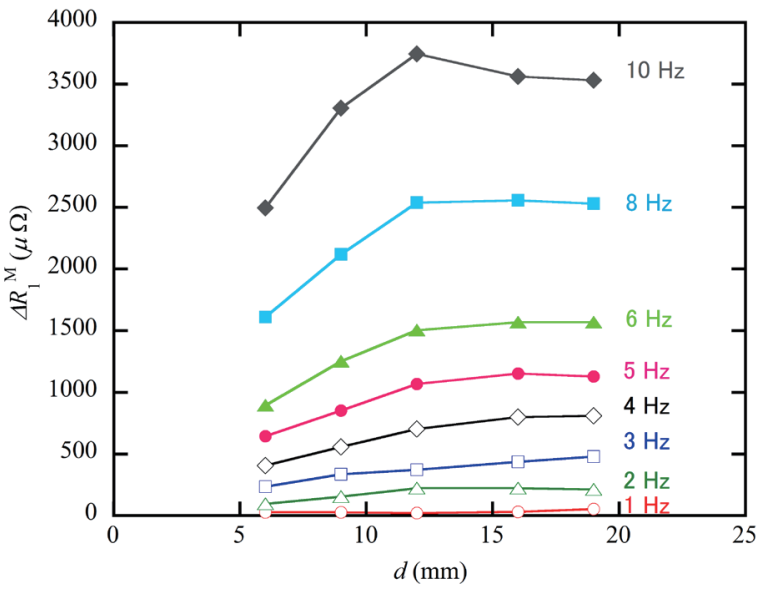

(a)

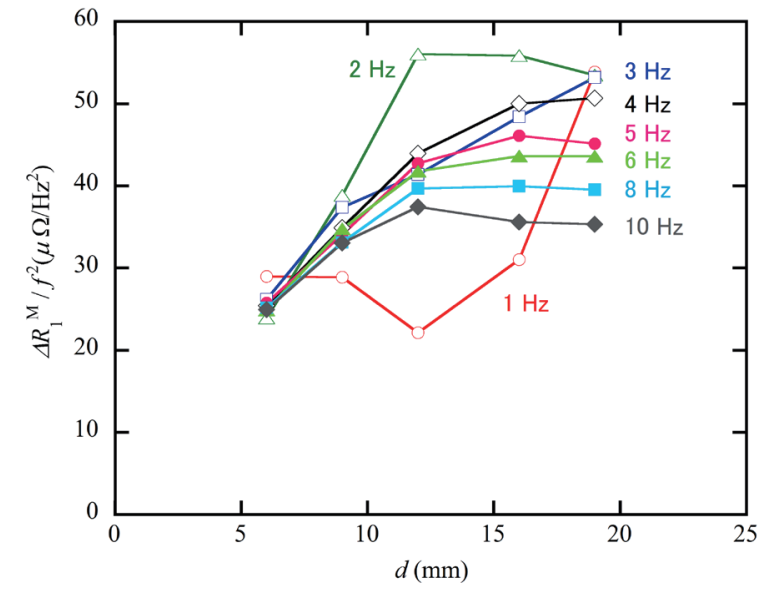

(b)

Fig. 4. (Color online) Relationship between the fundamental mutual equivalent resistance $\left(\Delta R_{1}^{M}\right)$ and the thickness of the steel plate $(d)$ when $f$ changes from 1 to $10 \mathrm{~Hz}$. (a) Resistance and (b) normalized resistance. 


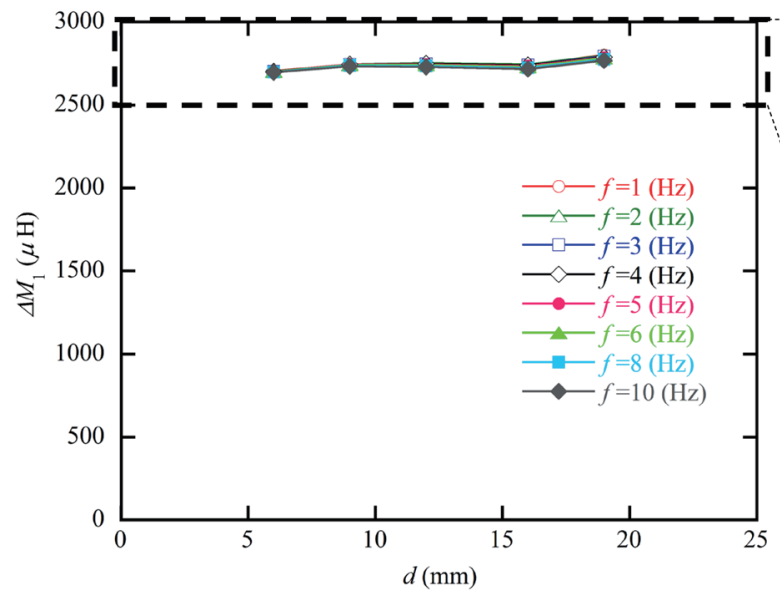

(a)

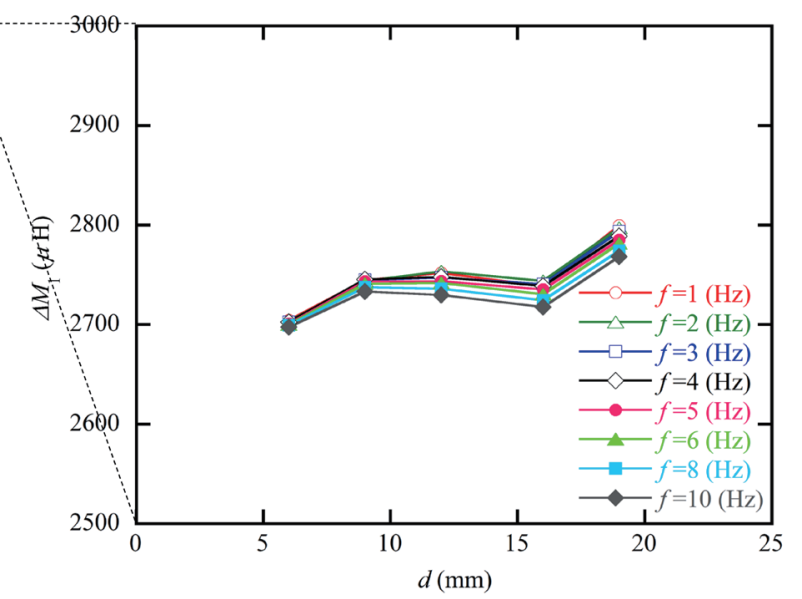

(b)

Fig. 5. (Color online) Relationship between the fundamental mutual inductance $\left(\Delta M_{1}\right)$ and the thickness of the steel plate $(d)$ when $f$ changes from 1 to $10 \mathrm{~Hz}$.

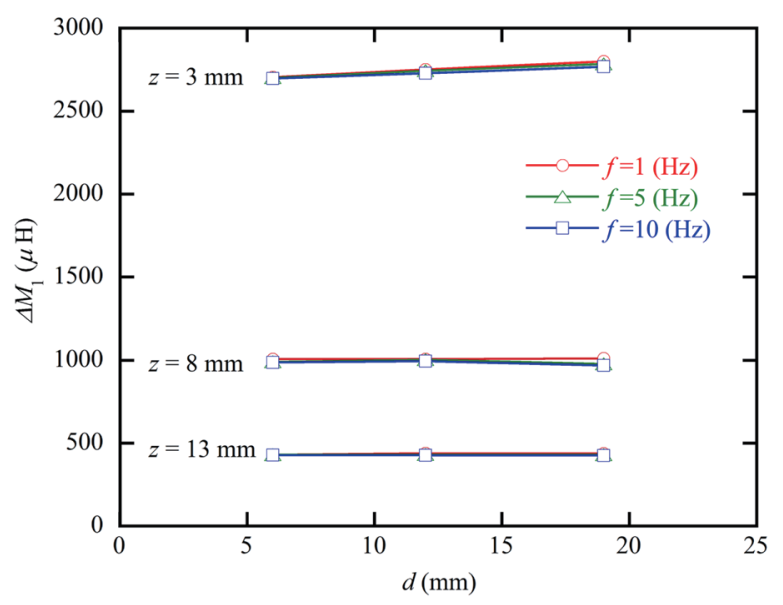

Fig. 6. (Color online) Relationship between the fundamental mutual inductance $\left(\Delta M_{1}\right)$ and the thickness of the steel plate $(d)$ the lift-off $z$ changes from 3 to $13 \mathrm{~mm}$.

Figure 7 shows the relationship between the normalized fundamental and harmonic equivalent mutual resistance $\left(\Delta R_{n}^{M} /(n f)^{2}\right)$ and $d$ when $f=1,2,3$, and $4 \mathrm{~Hz}$. The tendency of $R_{n}^{M} /(n f)^{2}(n>1)$ is almost the same as that of $\Delta R_{1}^{M} / f^{2}$. For example, when $n f \geq 4 \mathrm{~Hz}$, the skin effect appears, and $\Delta R_{1}^{M}$ monotonically increases in the domain $6 \leq d \leq 12 \mathrm{~mm}$.

\section{Discussion}

Firstly, we determined whether a square-wave inverter is applicable to ECT to estimate the thickness of the steel plate. The result shown in Fig. 4 demonstrates that $d$ can be estimated by measuring $\Delta R_{1}^{M}$ using a square-wave inverter if $f$ is appropriately chosen. This result 


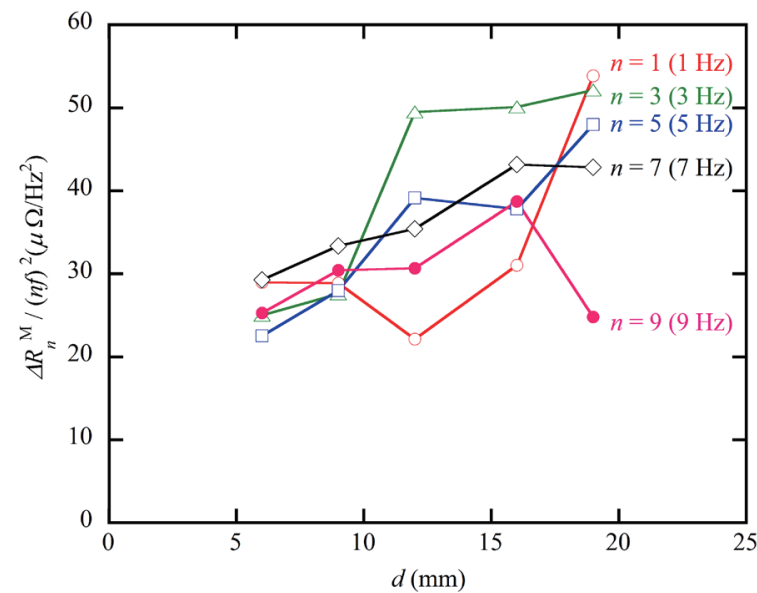

(a)

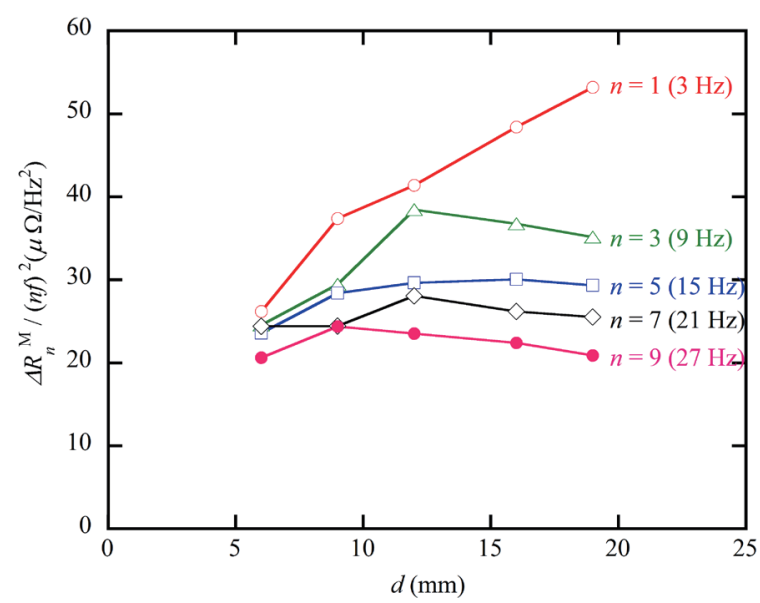

(c)

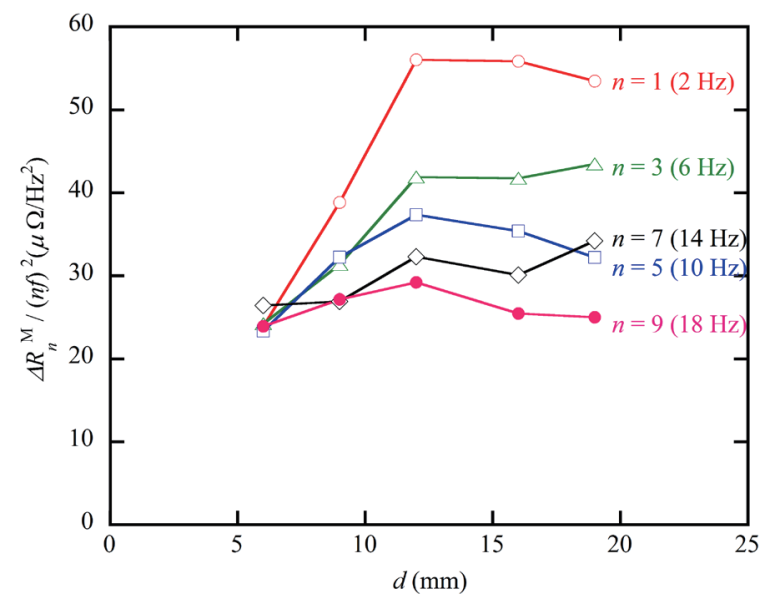

(b)

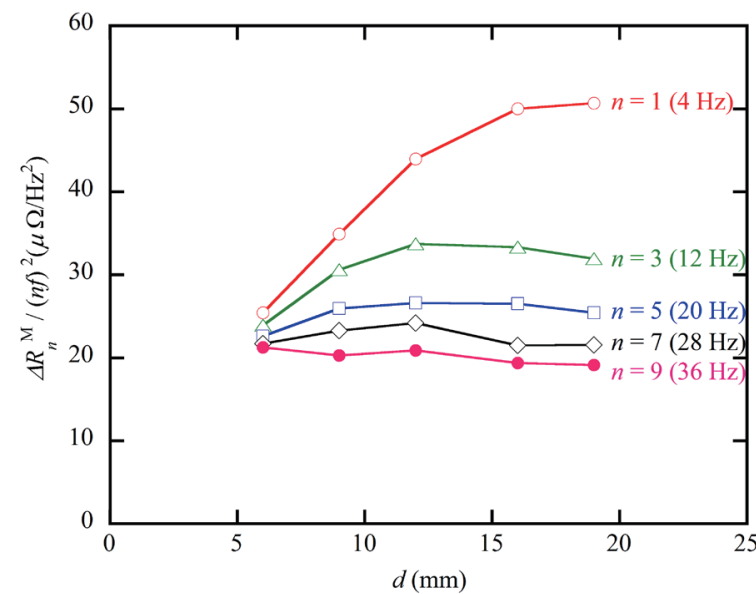

(d)

Fig. 7. (Color online) Relationship between the normalized fundamental and harmonic mutual equivalent resistance $\left[\Delta R_{n}^{M} /(n f)^{2}\right]$ and $d$ when $f=$ (a) 1 , (b) 2, (c) 3, and (d) $4 \mathrm{~Hz}$.

corresponds to that in a previous study when the sinusoidal wave is chosen for excitation. ${ }^{(8)}$ Therefore, the result of this study supports the validity of adopting a square-wave inverter for ECT instead of a linear amplifier.

Secondly, we determined whether more information can be obtained simultaneously from harmonic sinusoids as well as a fundamental sinusoid by using a square-wave inverter instead of a PWM inverter. The result shown in Fig. 7 demonstrates that the tendency of the harmonic signal is almost the same as that of the fundamental signal wiht the same frequency. This suggests that harmonic signals and a fundamental signal can be obtained simultaneously using a square excitation wave.

On the other hand, it has been reported that ECT enables us to obtain much information simultaneously using multifrequency data. ${ }^{(11-13)}$ In our future work, we will determine whether 
the harmonic signals generated by using the square excitation wave are applicable to obtaining much information simultaneously.

In this study, we chose a small excitation current for the experiment only to determine whether a square-wave inverter is applicable or not. In our future work, we will inspect the effectiveness of LF-ECT with a square-wave inverter when the lift-off is large.

\section{Conclusions}

In this study, we developed an LF-ECT system to determine whether a square-wave inverter is applicable to estimating the thickness of a steel plate. The result obtained by using the LFECT system demonstrates that a square-wave inverter is applicable to estimating the thickness by measuring the fundamental component of the mutual equivalent resistance. Moreover, we determined whether more information can be obtained simultaneously from harmonic sinusoids as well as a fundamental sinusoid. The result suggests that harmonic signals and a fundamental signal can be obtained simultaneously using a square excitation wave.

\section{Acknowledgments}

This work was supported in part by the Cross-Ministerial Strategic Innovation Promotion Program (SIP), Cabinet Office, Government of Japan.

\section{References}

1 J. Blitz: Electrical and Magnetic Methods of Non-Destructive Testing (Chapman \& Hall, London, 1997). https://doi.org/10.1007/978-94-011-5818-3

2 J. García-Martín, J. Gómez-Gil, and E. Vázquez-Sánchez: Sensors 11 (2011) 2525. https://doi.org/10.3390/ s110302525

3 T. Dogaru and S. T. Smith: IEEE Trans. Magn. 37 (2001) 3831. https://doi.org/10.1109/20.952754

4 K. Tsukada, T. Kiwa, T. Kawata, and Y. Ishihara: IEEE Trans. Magn. 42 (2006) 3315. https://doi.org/10.1109/ TMAG.2006.879754

5 F. Vacher, F. Alves, and C. Gilles-Pascaud: NDT and E Int. 40 (2007) 439. https://doi.org/10.1016/ j.ndteint.2007.02.002

6 K. Yao, T. Q. Yang, D. Yamasaki, K. Tazoh, and K. Enpuku: Jpn. J. Appl. Phys. 45 (2006) 4994. https://doi. org/10.1143/JJAP.45.4994

7 K. Allweins, G. Gierelt, H.-J. Krause, and M. V. Kreutzbruck: IEEE Trans. Appl. Supercond. 13 (2003) 250.

8 T. Sasayama, T. Ishida, M. Matsuo, and K. Enpuku: IEEE Trans. Appl. Supercond. 26 (2016) 9001305. https:// doi.org/10.1109/TASC.2016.2535366

9 T. Sasayama and K. Enpuku: Int. J. Appl. Electromagn. Mech. 59 (2019) 1275. https://doi.org/10.3233/JAE171117

10 T. Sasayama, Y. Yanamoto, S. Funaji, and T. Ao: IEEJ J. Ind. Appl. 4 (2015) 67. https://doi.org/10.1541/ ieejjia.4.67

11 D. F. He, M. Shiwa, J. P. Jia, J. Takatsubo, and S. Moriya: NDT and E Int. 44 (2011) 438. https://doi.org/10.1016/ j.ndteint.2011.04.004

12 W. Zhu, W. Yin, S. Dewey, P. Hunt, C. L. Davis, and A. J. Peyton, NDT and E Int. 86 (2017) 1. https://doi. org/10.1016/j.ndteint.2016.11.004

13 W. Yoshimura, T. Sasayama, and K. Enpuku: IEEE Trans. Magn. 55 (2019) 6201205. https://doi.org/10.1109/ TMAG.2019.2896590 


\section{About the Authors}

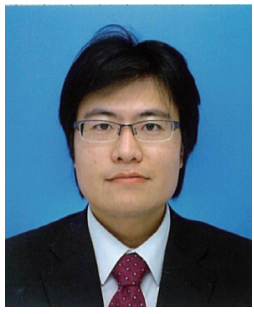

Teruyoshi Sasayama received his B. Eng., M. Eng., and Dr. Eng. degrees from Kyoto University, Japan, in 2007, 2009, and 2012, respectively. $\mathrm{He}$ is an associate professor in the Faculty of Information Science and Electrical Engineering, Kyushu University. His research interests are in the electromagnetic nondestructive testing and biomedical applications of magnetic nanoparticles. (sasayama@sc.kyushu-u.ac.jp)

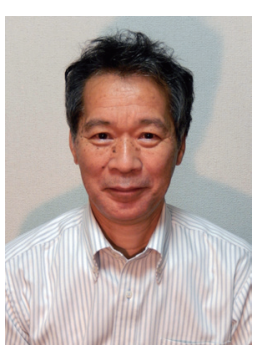

Keiji Enpuku received his B. Eng., M. Eng., and Dr. Eng. degrees from Kyushu University, Japan, in 1976, 1978, and 1981, respectively. $\mathrm{He}$ is a professor in the Research Institute of Superconductor Science and Systems, Kyushu University. He has been engaged in superconducting electronics. His current interest is in the development of a high-performance high-Tc SQUID magnetometer and its applications. (enpuku@sc.kyushu-u.ac.jp) 